\title{
Mechanical Behavior of Polystyrene Microgels
}

\author{
C. M. Roland* and P. G. Santangelo \\ Naval Research Laboratory, Chemistry Division, Code 6120, Washington, D.C. 20375-5342
}

\section{Antonietti and M. Neese ${ }^{\dagger}$}

Max Planck Institute of Colloids and Interfaces, Kantstrasse 55, D-14513 Tel tow-Sechof, Germany

Received November 17, 1998

\begin{abstract}
Polystyrene mi crogels are nanometer-sized particles produced by polymerization and crosslinking in microemulsion. As a result of their internal network structure, microgels have negligible intermolecular entanglement interactions. This can have a profound effect on their rheology; it also leads to glassy fracture resembling the behavior of low molecular weight, linear polystyrene. At higher frequencies, the mechanical response of microgels is quite similar to that of linear polystyrene. Breakdown of time-temperature superpositioning occurs in the softening zone of the viscoelastic spectrum. In the glass transition region, the segmental relaxation function is broadened and exhibits an enhanced dependence on temperature. The latter two effects, due to the cross-linking of the microgels, are also seen in conventional, macroscopic networks.
\end{abstract}

\section{Introduction}

Microgels are an interesting class of materials, having the internal structure of a macroscopic network, yet exhibiting viscous flow. ${ }^{1,2} \mathrm{~A}$ distinguishing feature is the absence of entanglements between different molecules (microgel particles), even for very high molecular weight. For polystyrene, there are about 170 monomer units between entanglements, ${ }^{3}$ which is more than twice the average number of units between cross-links for even the least cross-linked microgel studied herein. Since the microgel particles are spatially separated until after cross-linking, a high degree of cross-linking precludes intermolecular entanglements. Their unique morphology-densely packed, unentangled polyhedronsmarkedly affects the microgels' mechanical properties. ${ }^{1,2}$ Our efforts to characterize the rheology of PS microgels will be reported elsewhere; ${ }^{4}$ we restrict our attention herein to the mechanical behavior at higher frequencies.

\section{Experimental Section}

A detailed description of the microgel synthesis, as well as analysis of structure, has been published. ${ }^{5,6} \mathrm{~F}$ or the present study, various microgels were prepared by polymerization of styrene (AIBN initiator) in water, using myristyldiethanolamine- copper $^{7}$ or cetyltrimethylammonium chloride ${ }^{8}$ as the surfactant. Molecular weights, determined by light scattering, were from 280 to $50000 \mathrm{~kg} / \mathrm{mol}$ (corresponding to particle radii in the range $2.8-22 \mathrm{~nm}$ ). Network densities are determined by the amount of added m-diisopropenylbenzene; for the microgels herein, the number of cross-links per monomer unit, $f_{x}$, varied from $1 / 10$ tol/80.

Differential scanning cal orimetry (DSC) was carried out at $10 \mathrm{deg} / \mathrm{min}$ with a Perkin-EImer DSC-7. Dynamic mechanical spectra were obtained with a Bohlin VOR, using a parallel plate geometry. The plate size was adjusted according to the measurement temperature. Flexural stress/strain data were obtained at room temperature on strips $(\mathrm{I}=22.4 \mathrm{~mm}$; $\mathrm{w}=12$ $\mathrm{mm} ; \mathrm{t}=7 \mathrm{~mm}$ ) using an Instron 1135 . The maximum stress is calculated as

\footnotetext{
† Current affiliation: The Boston Consulting Group, Düsseldorf, Germany.
}

$$
\sigma=\frac{3 l}{2 \mathrm{wt}^{2}} \times \text { force }
$$

and the maximum strain is

$$
\epsilon=\frac{6 \mathrm{t}}{\mathrm{I}^{2}} \times \text { displacement }
$$

\section{Results and Discussion}

Stress-Strain Behavior. The extensibility of glassy polymers is modified by the presence of entangl ements. The crazing and fibril formation which accompanies drawing usually involves substantial chain disentanglement. ${ }^{9}$ This confers a molecular weight dependence to the strength of polymers, as noted long ago. ${ }^{10}$ Since microgels lack intermolecular entanglements, their deformation should mimic that of low molecular weight (unentangled) PS, rather than linear PS having comparable molecular weight.

This is illustrated in Figure 1, which shows the stress-strain response at room temperature for two microgels (having $f_{x}=1 / 10$ ) and for two linear PS. All polymers have comparable flexural moduli (=1.6 GPa), as seen in the figure. For linear PS, a $\mathrm{M}_{\mathrm{w}}$ substantially exceeding the entanglement molecular weight $(=18 \mathrm{~kg} /$ $\mathrm{mol}^{3}$ ) confers greater el ongation relative to that of the unentangled linear polymer $\left(\mathrm{M}_{\mathrm{w}}=24 \mathrm{~kg} / \mathrm{mol}\right)$. Such extensibility is absent in the microgels, even for an exceedingly high molecular weight of $50000 \mathrm{~kg} / \mathrm{mol}$. The stress-strain response of the microgels could be mistaken for that of unentangled linear PS.

Viscoelastic Softening Zone. It is well-known that a breakdown of time-temperature superpositioning occurs in the softening zone of the viscoel astic spectrum. First discovered for polystyrene, ${ }^{11}$ this phenomenon results from the different temperature dependences of the chain motions in comparison to the more local segmental dynamics. ${ }^{12-15}$ The latter have larger time/ temperature shift factors, which means that at lower temperature their contribution to the spectrum is less separated from that of the chain modes. In the softening 


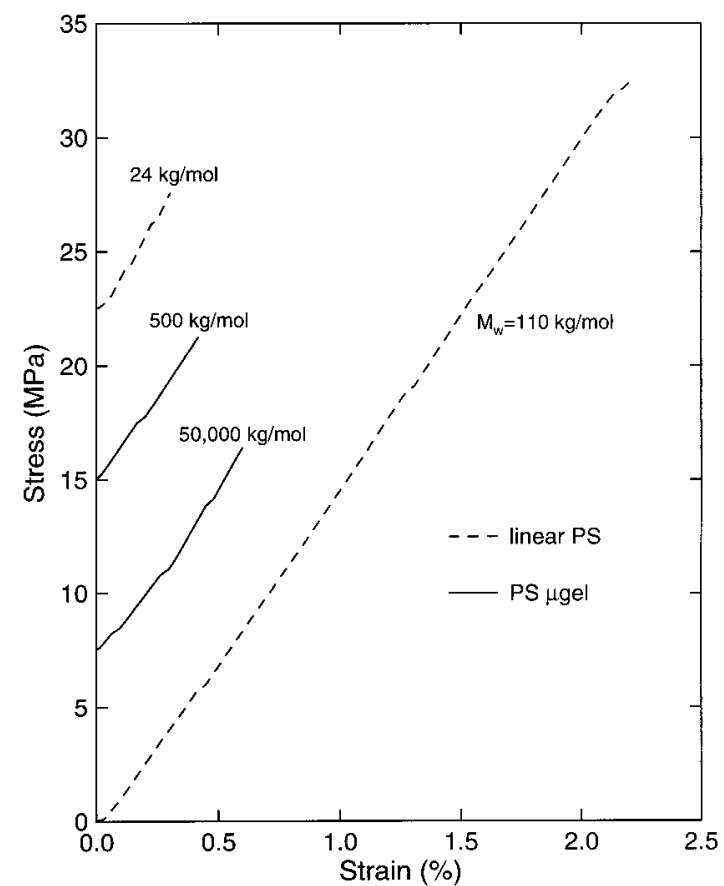

Figure 1. Bending stress versus flexural strain (at room temperature) for linear and mi crogel polystyrenes having the indicated molecular weight. The curves are displaced vertically for clarity.

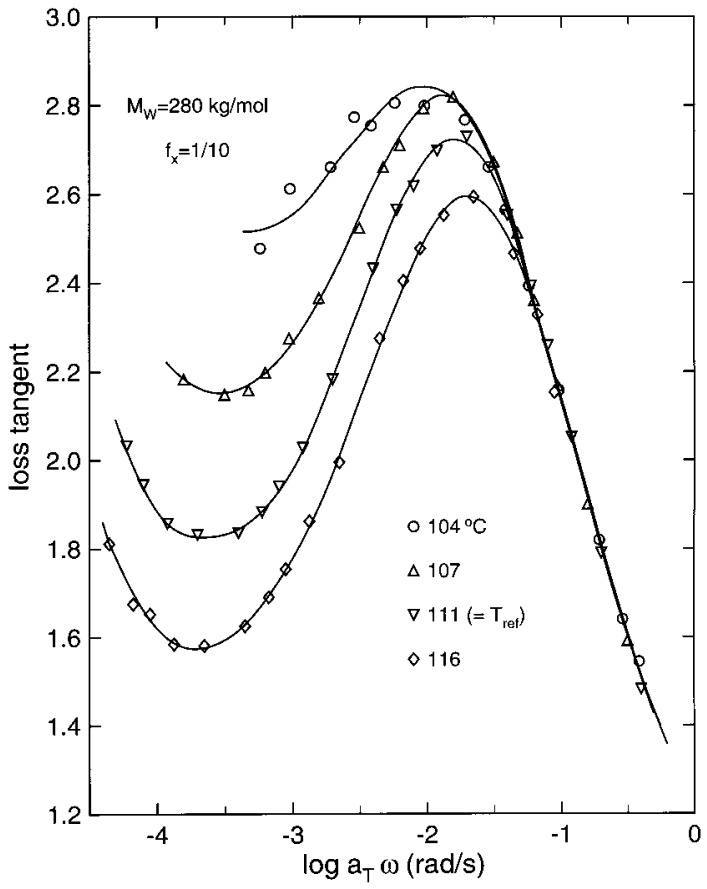

Figure 2. Temperature dependence of loss tangent for PS microgel, revealing the breakdown of time-temperature superpositioning in the softening zone. The curves were shifted horizontally to superimpose at higher frequency.

zone, where both modes contribute, their different temperature dependences cause thermorheol ogical complexity.

Displayed in Figure 2 is the loss tangent measured for a PS microgel measured at four different temperatures. While shifting the data brings the high-frequency side of the peaks into coincidence, the peaks measured at lower temperature are broader, with shallower minima. These characteristics are very similar to those observed for linear PS. ${ }^{16}$

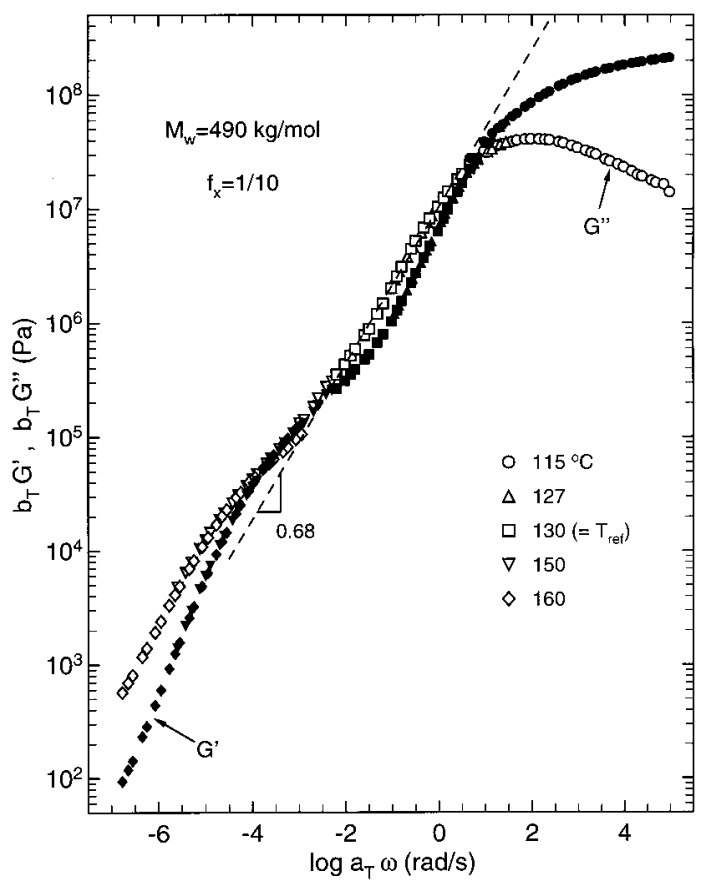

Figure 3. Master curves of storage and loss moduli for PS microgel. The superpositioning is only approximate (see Figure 2). The dashed line indicates the power-law behavior observed in the softening zone; fitting the data at $130{ }^{\circ} \mathrm{C}$ yields a slope $=0.68$.

Figure 3 shows dynamic mechanical master curves for the storage and loss moduli of a PS microgel. The detailed shape is only approximate, given the aforementioned breakdown of time-temperature superpositioning in the transition zone. Nevertheless, the curves closely resemble the spectra observed for linear PS, when the latter has a molecular weight about an order of magnitude smaller than that of the microgel in Figure 3. ${ }^{17}$

At $130^{\circ} \mathrm{C}$ the measured data fall within the power law regi on of the softening zone. Using only data points in Figure 3 obtained at this temperature, we can calculate the slope, $d \log \left(G^{\prime \prime}(\omega)\right) / d \log (\omega)$. The negative inverse of this quantity is referred to as the "steepness index" in the damped lattice model of Tobolsky and Aklonis. ${ }^{18,19}$ This sl ope al so corresponds approximately to the high-frequency exponent of the BSW spectrum..$^{20}$ The value determined for the microgel, $\mathrm{d} \log \left(\mathrm{G}^{\prime \prime}(\omega)\right) / \mathrm{d}$ $\log (\omega)=0.68$, is identical to the value reported for linear PS.21 It is smaller (i.e., sharper transition, larger steepness index) than that of other polymers. ${ }^{12,21}$

Segmental Relaxation. At higher frequencies in Figure 3 there is a dispersion in the loss modulus, reflecting local segmental relaxation ( $\alpha$-transition). In Figure 4, this peak is shown on a linear ordinate scale for both linear PS and four microgels of varying crosslink density $\left(1 / 80 \leq f_{x} \leq 1 / 10\right)$. These microgel s all have approximately the same molecule weight (ca. $10^{3} \mathrm{~kg} /$ mol). Their peak breadth is greater than for linear PS and systematically broadens with increasing cross-link density. This is expected, since the reduction in the available configurations due to the network structure gives rise to enhanced cooperativity among segments. Stronger intermolecular cooperativity is known to broaden the relaxation function of networks. ${ }^{22-26} \mathrm{~N}$ ote that a distribution of strand lengths between cross-links would also broaden the relaxation function; however, the uniform distribution of the cross-linking agent 


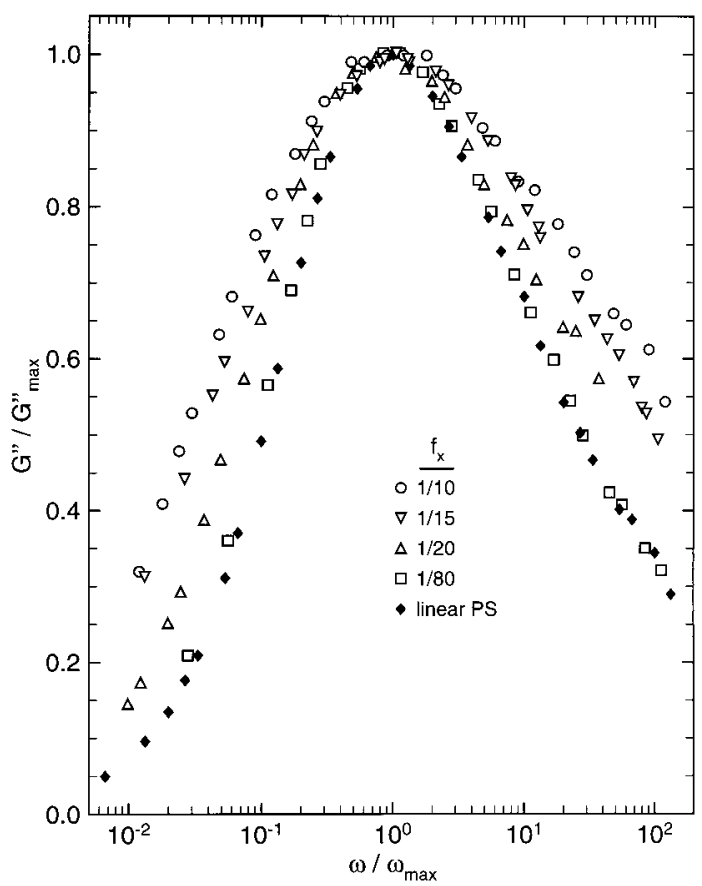

Figure 4. Segmental relaxation dispersions for a linear PS $\left(\mathrm{M}_{\mathrm{w}}=90 \mathrm{~kg} / \mathrm{mol}\right)$ and microgels having the indicated degree of cross-link (with $M_{w} \geq 10^{3} \mathrm{~kg} / \mathrm{mol}$ ). The curves have been shifted to superimpose at their peaks, which reveals the increasing breadth of the relaxation function with increased cross-linking of the microgel.

within the polymerizing microgel, as well as the favorable copolymerization parameters, ${ }^{27}$ makes such an effect unlikely.

There is an additional influence on the shape of the relaxation function for networks, inhomogeneous broadening due to a distribution of relaxation behaviors. The latter arises from the varying degree to which segments, in different proximity to a network junction, have their local dynamics constrained by the junction. The extent of this inhomogeneous broadening is in proportion to the concentration of cross-links. ${ }^{24}$ Both these effectsenhanced intermolecular cooperativity and inhomogeneous broadening-are presumably operative in the microgels.

A "most probable" relaxation time for local segmental motion can be defined as the inverse of the frequency of the loss modulus maximum, $\tau \equiv 1 / \omega_{\text {peak }}$ I In Figure 5 we plot these $\tau$ as measured for the polystyrenes at various temperatures. The data were fitted to the Vogel-Fulcher equation ${ }^{3}$

$$
\tau=\mathrm{a} \exp \left(\frac{\mathrm{b}}{\mathrm{T}-\mathrm{T}_{\infty}}\right)
$$

At fixed temperature, there is a systematic increase in the magnitude of $\tau$ with increasing cross-link density. This trend is consistent with increased intersegmental constraints on the local relaxation, as inferred from the peak breadths in Figure 4. Such behavior, of course, also underlies the increase in glass transition temperature with cross-linking. 26,28

The breadths of the peaks in Figure 4 are expected to correlate with the effect temperature has on the relaxation times (at least that portion of the breadth not due to inhomogeneous broadening medhanisms). ${ }^{14,24,29}$ As demonstrated for many polymers and small molecule glass formers, relaxation times associated with broader

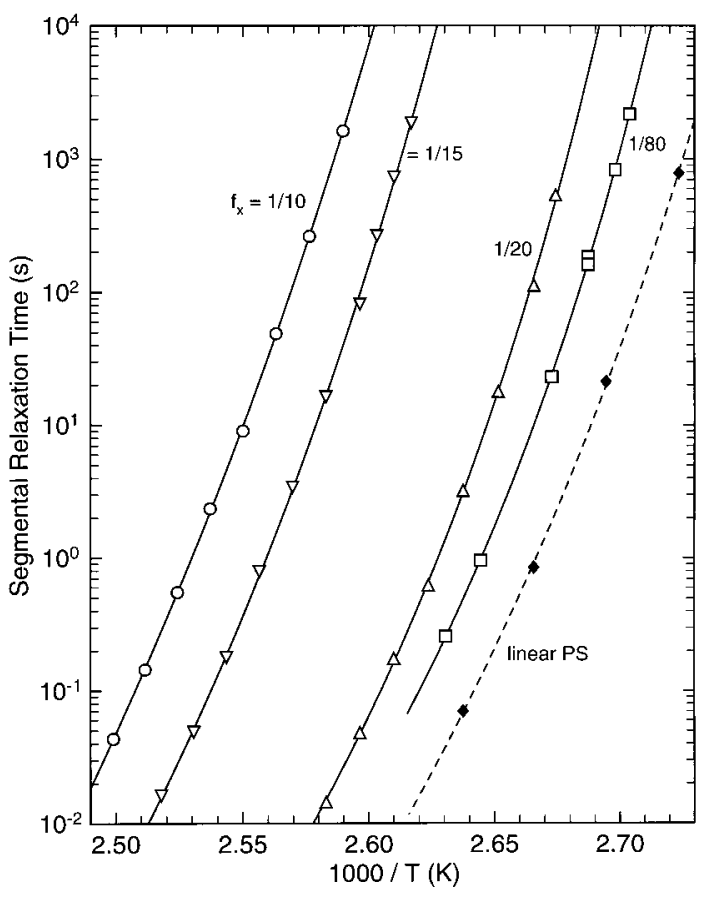

Figure 5. Arrhenius plots of local segmental relaxation times for the linear and microgel PS shown in Figure 4.

peaks depend more strongly on temperature. ${ }^{30-34}$ This effect is usually demonstrated by comparison of fragilities, defined as the slope of an Arrhenius plot of relaxation times in which temperature is normalized by some reference temperature. ${ }^{35-37}$ In terms of the VogelFulcher parameters, fragility can be expressed as ${ }^{38}$

$$
\left.\frac{\mathrm{d} \log (\tau)}{\mathrm{d}\left(\mathrm{T}_{\mathrm{ref}} f \mathrm{~T}\right)}\right|_{\mathrm{T}_{\mathrm{T}} \mathrm{T}_{\text {ref }}}=\frac{\mathrm{b}}{\ln (10) \mathrm{T}_{\text {ref }}}\left(1-\mathrm{T}_{\infty} / \mathrm{T}_{\mathrm{ref}}\right)^{-2}
$$

where $T_{\text {ref }}$ is the temperature at which the relaxation time equals an arbitrary value; for mechanical data, typically $\tau\left(\mathrm{T}_{\text {ref }}\right)=100 \mathrm{~s}$.

Fragilities obtained in this manner for the various microgels are displayed in Figure 6. There is no significant trend with cross-link density; the average fragility equals 158. However, the microgels are all substantially more "fragile" than linear PS. The fragility of linear PS is molecular weight dependent, attaining a value as high as 146 at the highest molecular weights. ${ }^{39}$ The fragility of polymers in general spans a range of roughly 40$200.30,34$

The quantity used as the abscissa in Figure 6 is the heat capacity increment at $\mathrm{T}_{\mathrm{g}}$. This form of displaying the data is drawn from Angell's energy landscape interpretation of fragility, ${ }^{35-37}$ which predicts a proportionality between fragility and $\Delta \mathrm{C}_{\mathrm{p}}\left(\mathrm{T}_{\mathrm{g}}\right)$. According to Angell, $35-37$ the strength or fragility of relaxation be havior reflects the topology of the potential energy hypersurface governing diffusions, reorientations, and, for the case of polymers, segmental reconfigurations. Fragile liquids are associated with potential surfaces having a high density of minima, and hence such liquids exhibit high configurational heat capacity changes at $T_{g}, \Delta C_{p}\left(T_{g}\right)$. According to this interpretation, when a fragile glass is warmed through the glass transition, a wide variety of structural arrangements become available, engendering substantially reduced relaxation times. Strong liquids would show the opposite behavior; having potential energy surfaces with few minima and thus 


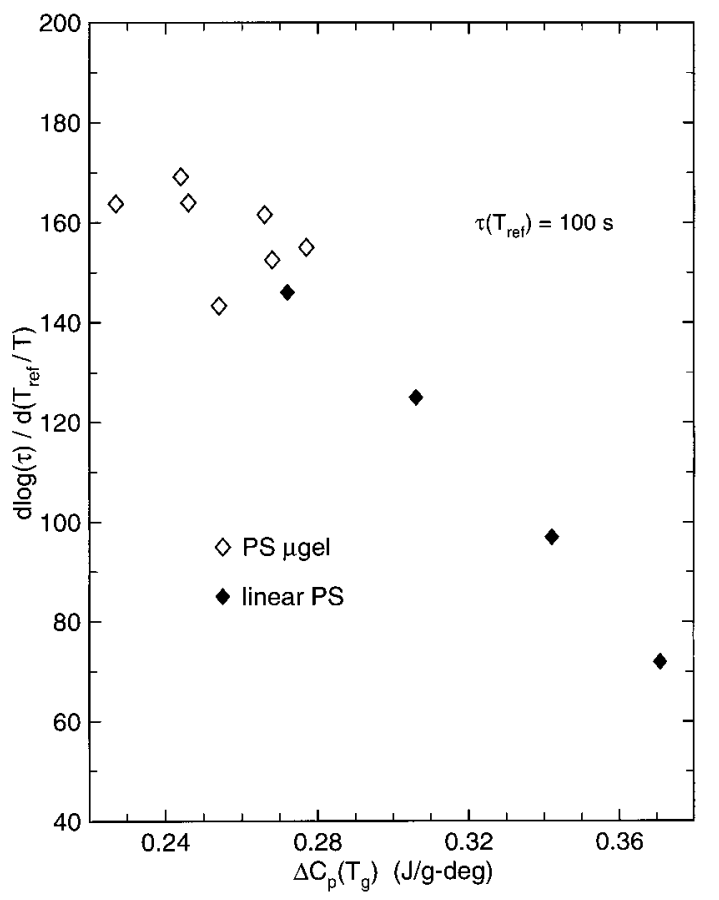

Figure 6. Fragility of linear and microgel PS (calculated from eq 4) as a function of the heat capacity increment at $T_{g}$ measured calorimetrically.

small $\Delta C_{p}\left(T_{g}\right)$, their relaxation times would be less sensitive to temperature changes. Many small molecule glass-forming liquids conform to this model. ${ }^{35-37}$

The relationship expected from Angell's model is not observed in Figure 6. Higher cross-linking reduces the heat capacity increment at $T_{g}$, consistent with the reduction in degrees of freedom accompanying crosslinking. However, the smaller $\Delta C_{p}\left(T_{g}\right)$ is not accompanied by a significant alteration of the fragility. Moreover, as seen in Figure 6, and reported previously, ${ }^{39}$ the relationship between fragility and $\Delta C_{p}\left(T_{g}\right)$ for linear PS is opposite to the theoretical prediction. Parenthetically, we note that the behavior of conventional polymer networks is similarly at odds with the Angell model. ${ }^{26}$

Since interparticle interactions are weak for the unentangled microgels, the mechanical properties are clearly dominated by their internal structure. Nevertheless, packing density or long-range coupling may exert some influence on the microgel behavior. Figure 7 displays the segmental relaxation times measured for three microgels of varying molecular weight (i.e., particle size); all have $\mathrm{f}_{\mathrm{x}}=10 \%$. There is a systematic increase in $\tau$ as particle size is reduced. This would suggest more cooperative local relaxation; however, particle size has a negligible effect on the temperature dependence of these segmental relaxation times. The slower relaxation of smaller particles may be the consequence of closer packing and consequently more steric interference or perhaps the population of otherwise inactive long-range coupling modes.

\section{Summary}

The mechanical behavior of the microgels described herein is in most respects quite similar to that of linear polystyrene, at least when the latter is of relatively low molecular weight. A distinguishing feature of the structure of these high molecular weight microgels-the absence of entanglement couplings between moleculeshas a negligible effect on the local segmental dynamics.

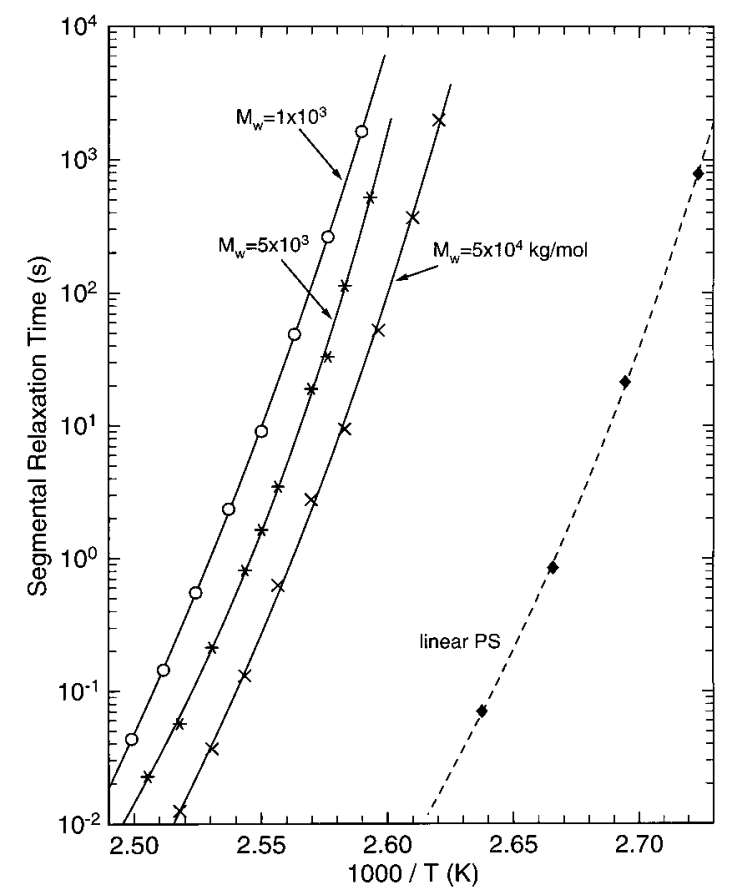

Figure 7. Arrhenius plots of the local segmental relaxation times of microgels having $\mathrm{f}_{\mathrm{x}}=1 / 10$ and the indicated molecular weights. The latter correspond to particle radii equal to 7.6 (O), $12.5(*)$, and $22 \mathrm{~nm}(\times)$, respectively, as determined by light scattering. The linear PS has a molecular weight equal to $90 \mathrm{~kg} / \mathrm{mol}$.

However, since segmental relaxation is manifested over a substantial length scale, ${ }^{40-44}$ cross-link junctions constrain the motion of even topological ly remote chain segments. Consequently, segmental relaxation times, as well as the shape of the relaxation function itself, are affected by cross-link density. This extended range of influence of network junctions has an analogous effect on crystallization in networks. ${ }^{45-47}$

Along with the study of segmental dynamics in polymers, microgels offer the possibility of serving as model networks for the study of rubber elasticity. ${ }^{48}$ This is an area in which the role of entanglements remains to be quantitatively established, and hence any insights microgels can provide will be welcome.

Acknowledgment. The work at NRL was supported by the Office of Naval Research. C. A. Bero contributed to the early work while a Naval Research Lab/National Research Council postdoctoral fellow.

\section{References and Notes}

(1) Antonietti, M.; Pakula, T.; Bremser, W. Macromol ecules 1995, 28, 4227.

(2) Roland, C. M.; Bero, C. A.; Ngai, K. L.; Antonietti, M. Mater. Res. Soc. Proc. Ser. 1996, 411, 367.

(3) Ferry, J . D. Viscoelastic Properties of Polymers; Wiley: New York, 1980.

(4) Santangelo, P. G.; Roland, C. M.; Antonietti, M.; Neese, M., to be published.

(5) Antoniettii, M.; Basten, R.; Lohmann, S. Macromol. Chem. Phys. 1995, 196, 441.

(6) Antonietti, M.; Bremser, W.; Schmidt, M. Macromolecules 1990, 23, 3796.

(7) Antonietti, M.; Nestl, T. Macromol. Rapid Commun. 1994, $15,111$.

(8) Antonietti, M.; Bremser, W.; Muschenborn, D.; Rosenauer, C.; Schupp, B.; Schmidt, M. Macromol ecules 1991, 24, 6636.

(9) Polymer Fracture, 2nd ed.; Springer: New York, 1987.

(10) Flory, P. J . J . Am. Chem. Soc. 1945, 67, 2048.

(11) Plazek, D. J.J . Phys. Chem. 1965, 6, 612. 
(12) Ngai, K. L.; Plazek, D. J . Rubber Chem. Technol. 1995, 68, 376.

(13) Plazek, D. J.; Chay, I.-C.; Ngai, K. L.; Roland, C. M. Macromol ecules 1995, 28, 6432.

(14) Santangelo, P. G.; Ngai, K. L.; Roland, C. M. Macromolecules 1996, 29, 3651.

(15) Santangelo, P. G.; Roland, C. M. Macromolecules 1998, 31, 3715.

(16) Cavaille, J . Y.; J ordan, C.; Perez, J .; Monnerie, L.; J ohari, G. P. J . Polym. Sci., Polym. Phys. Ed. 1987, 25, 1235.

(17) Schausberger, A.; Schindlauer, G.; J aneschitz-Kriegl, H. Rheol. Acta 1985, 24, 220.

(18) Tobolsky, A. V.; Aklonis, J I . J . Phys. Chem. 1964, 68, 1970.

(19) Aklonis, J. J .; Rele, V. B. J . Polym. Sci. 1974, C46, 127.

(20) J ackson, J. K.; De Rosa, M. E.; Winter, H. H. Macromolecules $1994,27,2426$

(21) Aklonis, J. J .; Macknight, W. J . Introduction to Polymer Viscoelasticity, 2nd ed.; Wiley: New York, 1983; p 176.

(22) Ngai, K. L.; Roland, C. M.; Yee, A. F. Rubber Chem. Technol. 1993, 66, 817

(23) Ngai, K. L.; Roland, C. M. Macromolecules 1994, 27, 2454

(24) Roland, C. M. Macromolecules 1994, 27, 4242.

(25) Roland, C. M.; Ngai, K. L.; Plazek, D. J . J . Comput. Theor. Polym. Sci. 1997, 7, 133

(26) Santangelo, P. G.; Roland, C. M. Phys. Rev. B 1998, 58, 14121.

(27) Hild, G.; Okasha, R. Makromol. Chem. 1985, 186, 389.

(28) Nielsen, L. E. Mechanical Properties of Polymers and Composites; Marcel Dekker: New York, 1974; Vol. 1.

(29) Roland, C. M. Macromolecules 1992, 25, 7031.
(30) Plazek, D. J .; Ngai, K. L. Macromolecules 1991, 24, 1222.

(31) Roland, C. M.; Ngai, K. L. Macromolecules 1991, 24, 5315; 1992, 25, 1844.

(32) Roland, C. M.; Ngai, K. L. Macromolecules 1992, 25, 5765.

(33) Ngai, K. L.; Roland, C. M. Macromolecules 1993, 26, 6824

(34) Bohmer, R.; Ngai, K. L.; Angell, C. A.; Plazek, D. J . J . Chem. Phys. 1993, 99, 4201.

(35) Angell, C. A. J . Non-Cryst. Solids 1991, 131-133, 13.

(36) Angel, C. A. Science 1995, 267, 1924.

(37) Angell, C. A.; Poole, P. H.; Shao, J . Nuovo Cimento 1994, 16, 883.

(38) Hodge, I. M. J . Non-Cryst. Solids 1996, 202, 164.

(39) Santangelo, P. G.; Roland, C. M. Macromolecules 1998, 31, 4581.

(40) Read, B. E. Polym. Eng. Sci. 1983, 23, 835

(41) Adolf, D. B.; Ediger, M. D. Macromolecules 1992, 25, 1074.

(42) Bahar, I.; Erman, B.; Kremer, F.; Fischer, E. W. Macromolecules 1992, 25, 816

(43) Santangelo, P. G.; Ngai, K. L.; Roland, C. M. Macromolecules 1993, 26, 2682.

(44) Mott, P. H.: Roland, C. M. Macromolecules 1998, 31, 7095.

(45) Kuhn, W.; Majer, J. Angew. Chem. 1956, 68, 345.

(46) Roberts, D. E.; Mandelkern, L. J . Am. Chem. Soc. 1960, 82, 1091.

(47) Roland, C. M.; Buckley, G. S. Rubber Chem. Technol. 1991 $64,74$.

(48) Antonietti, M. Angew. Chem., Int. Ed. Engl. 1988, 27, 1743. MA9817828 